\title{
Distribución de los Patrones Venosos de la Fosa Cubital en una Muestra de Personas Nacidas en Bucaramanga, Colombia
}

\author{
Distribution Pattern of the Veins of the Cubital Fossa \\ in a Sample of People Born in Bucaramanga, Colombia
} "Edgar Giovanni Corzo Gómez; *** Olga Lucía Gómez Díaz; ${ }^{* * *}$ María Eugenia Niño Mantilla;
***** Ruby Jackeline Rey Triana \& ${ }^{* * * *}$ Leidy Johanna Pedraza Díaz

CORZO, G. E. G; GÓMEZ, D. O. L.; NIÑO, M. M. E.; REY, T. R. J. \& PEDRAZA, D. L. J. Distribución de los patrones venosos de la fosa cubital en una muestra de personas nacidas en Bucaramanga, Colombia. Int. J. Morphol., 28(4):1011-1018, 2010.

RESUMEN: El uso racional del acceso a las venas superficiales en la fosa cubital es de especial relevancia en pacientes pediátricos, con fístulas para diálisis, en quimioterapia, o como vías para acceso venoso central. Se han descrito varios patrones venosos con sus beneficios o riesgos. Se determinó la frecuencia de los patrones venosos en una población de ambos sexos nacida en Bucaramanga, Colombia, en 800 miembros superiores (200 hombres y 200 mujeres), conforme a la clasificación propuesta por del Sol et al. (1988, 2007). El análisis descriptivo para variables cualitativas nominales y ordinales mediante porcentajes, las cuantitativas con media y desviación estándar en el caso de variables con distribución normal y las no paramétricas identificadas con número mediante mediana y rango intercuartil. Se comparó género y patrón venoso mediante la prueba de Kwallis y chi cuadrado. El patrón predominante fue el III, 297 casos (37\%) determinado por una alta frecuencia en mujeres (54\%), seguido del II 190 casos (24\%). En el lado derecho para ambos géneros el patrón más frecuente fue el III, 141 casos (35\%), seguidos del II, 89 casos (22\%). En el lado izquierdo para ambos géneros predominó el patrón III, 156 casos (39\%), seguido por el II con 101 casos (25\%). El patrón II fue el mayor en hombres (28\%), seguido del III (21\%). En mujeres predominó el patrón III, seguido del II (19\%). En el lado derecho en hombres, predominó el II (24\%) seguido del IV (23\%). En el lado derecho en mujeres predominó el III (51\%) seguido del II (20\%). En el lado izquierdo en hombres, predominó el patrón II (32\%) seguido del III (21\%). En el lado izquierdo en mujeres predominó III (57\%) seguido del II (18\%). El patrón que en más ocasiones se presentó al mismo tiempo en ambos lados en la misma persona fue el III $(23,5 \%)$.

PALABRAS CLAVE: Punción venosa; Venas; Fosa cubital.

\section{INTRODUCCIÓN}

La canalización de las venas superficiales del miembro superior es uno de los más frecuentes procedimientos invasivos que generan malestar y dolor asociados a riesgo de infección o hematoma. Horowitz (2001) señaló el riesgo de lesión de los nervios cutáneos antebraquiales lateral y medial, al igual que la rama superficial del nervio radial generando en ciertas ocasiones la aparición de dolor neuropático y limitación funcional.

Vialle et al. (2001) resaltaron el riesgo de lesión de la rama superficial del nervio radial durante la inserción del catéter en la porción distal del antebrazo en la canalización de la vena cefálica; mostraron que en 33 cadáveres estudiados, la rama sensitiva del nervio radial inicia un curso subcutáneo a una distancia promedio de 8,16 centímetros medidos desde el proceso estiloideo del radio. En cada muestra el nervio cruzó la vena cefálica o una de sus afluentes al menos una vez, y en 14 de los 33 casos, la vena y el nervio se cruzaban en 2 sitios diferentes. Abrams et al. (1992) y Auerbach et al. (1994) reportaron distancias promedio similares (9 y 8,6 centímetros, respectivamente).

Médico, Maestría en Ciencias Básicas Biomédicas. Coordinador Académico del Programa de Medicina, Facultad de Ciencias de la Salud, Universidad Autónoma de Bucaramanga. Bucaramanga, Colombia.

** Especialista en administración de servicios de salud. Especialista en relaciones laborales. Directora del programa de Enfermería de la Universidad Autónoma de Bucaramanga. Bucaramanga, Colombia.

*** Médico, Maestría en Epidemiología. Profesor asociado, Facultad de Ciencias de la Salud, Universidad Autónoma de Bucaramanga. Bucaramanga, Colombia.

***** Estudiante, Programa de Enfermería. Universidad Autónoma de Bucaramanga. Bucaramanga, Colombia. 
Stahl et al. (1996), Thrush \& Belsole (1995) y Boeson et al. (2000) reportaron casos de neuromas o neuritis de la rama sensitiva del nervio radial luego de la canalización de la vena cefálica.

Yamada et al. (2008) estudiaron 128 miembros superiores de cadáveres y encontraron que en el $21 \%$ de los casos el nervio cutáneo antebraquial medial cursaba sobre las venas mediales de la fosa cubital generando riesgo de punción al canalizar estas venas; determinaron que la canalización de la vena intermedia cefálica es la que ofrece menor riesgo de lesión nerviosa.

Lirk et al. (2004) encontraron que en 2 de 26 miembros superiores disecados existía una arteria radial accesoria de ubicación superficial cercana a los sitios de canalización de la vena cefálica, lo cual genera un riesgo de punción arterial durante el acceso venoso; aunque la frecuencia de esta arteria superficial accesoria ha sido reportada con una frecuencia de 0,5-1\% por Rodriguez-Niedenfuhr et al. (2001); se ha recomendado asumir la costumbre de palpar el sitio de canalización de la vena cefálica en búsqueda del pulso de esta arteria si existiera.

Por estas razones, el conocimiento de la terminología de los patrones venosos de la fosa cubital y la determinación de la frecuencia de estos patrones en algunos grupos poblacionales se convierte en una herramienta esencial en la práctica diaria de ciertos profesionales de la salud como los enfermeros, los anestesiólogos y en particular en el área de pediatría por las características propias de los miembros superiores y las venas en los niños.

Para el estudio de venas superficiales de niños entre 0 y 1 año, del Sol et al. (1988) emplearon una clasificación de los patrones venosos que ha guiado otros estudios posteriores y han permitido correlacionar y poner en contexto estudios previos que utilizaban terminología variada para nombrar las venas. Estos patrones se estudiaron manteniendo la terminología por del Sol et al. (1990) en 400 antebrazos de jóvenes chilenos de ambos sexos (57\% hombres) y luego por del Sol et al. (2007) en 150 personas del grupo étnico Mapuche en Chile (30 hombres y 120 mujeres).

En este proceso se excluyó a la clásica distribución en "M" de ser uno de los patrones principales a tener en cuenta en razón a que su frecuencia de hallazgo fue muy baja, 0,5\% encontrada en 1990 por del Sol et al. en el análisis de las venas superficiales de 200 jóvenes chilenos de ambos sexos, y 1\% encontrado en 2007 por del Sol et al. en 300 miembros superiores del grupo étnico Mapuche en Chile. Estos valores de frecuencia para el patrón clásico en "M" concuerdan con los hallazgos de Okamoto (1922) encon- trando un $1 \%$ de esta distribución en 200 hombres japoneses; al igual que el 1,7\% encontrado por Skewes (1944) en 140 hombres chilenos de ascendencia hispánica y Halim \& Abdi (1974) quienes señalaron esta distribución con un 6,5\% en hindúes. Los valores descritos por estos autores se contraponen con los hallazgos de Paturet (1951) quien describe esta distribución venosa en el $60 \%$ de los casos o Singh et al. (1982) quienes informan un $40 \%$ de hallazgo en un estudio de 300 personas nigerianas.

El presente estudio busca establecer la frecuencia de presentación de estos patrones en una población de personas de ambos sexos nacidas en la ciudad de Bucaramanga, Colombia.

\section{MATERIAL Y MÉTODO}

Se estudiaron 800 miembros superiores de personas entre los 18 y 46 años (200 hombres y 200 mujeres), Cada participante recibió una explicación completa del procedimiento a realizar y su aceptación a participar quedó consignada en la firma de un consentimiento informado que señalaba el mínimo riesgo al que se sometía cada participante.

El procedimiento incluyó que con la persona sentada y el miembro superior en una superficie firme y plana se aplicó calor local en cada antebrazo con una compresa conectada a un paquete eléctrico a una temperatura entre 36 y 45 grados centígrados durante 10 minutos y posteriormente se aplicó un torniquete de velcro en el tercio medio del brazo. Dos evaluadores de forma independiente visualizaron y clasificaron el patrón de las venas superficiales a nivel de la fosa cubital; si no coincidía la clasificación se repetía el procedimiento y se incluía la opinión de un tercer evaluador. Concomitantemente se realizaron registros fotográficos del patrón venoso de cada miembro superior. Se excluyeron los participantes en quienes no se evidenció con claridad la distribución venosa superficial y en los que no coincidió la clasificación de los evaluadores independientes en la segunda oportunidad.

Los patrones venosos establecidos fueron adaptados a partir de los estudios de del Sol et al. $(1988,2007)$ y son descritos a continuación:

Tipo I: La vena cefálica (VC) se divide en vena intermedia basílica (VIB) y vena intermedia cefálica (VIC), uniéndose a la vena basílica (VB) y vena cefálica accesoria (VCA) respectivamente. La vena intemedia basílica (VIB) era de mayor calibre y la vena intermedia del antebrazo (VIA) drenaba generalmente en la VB. 


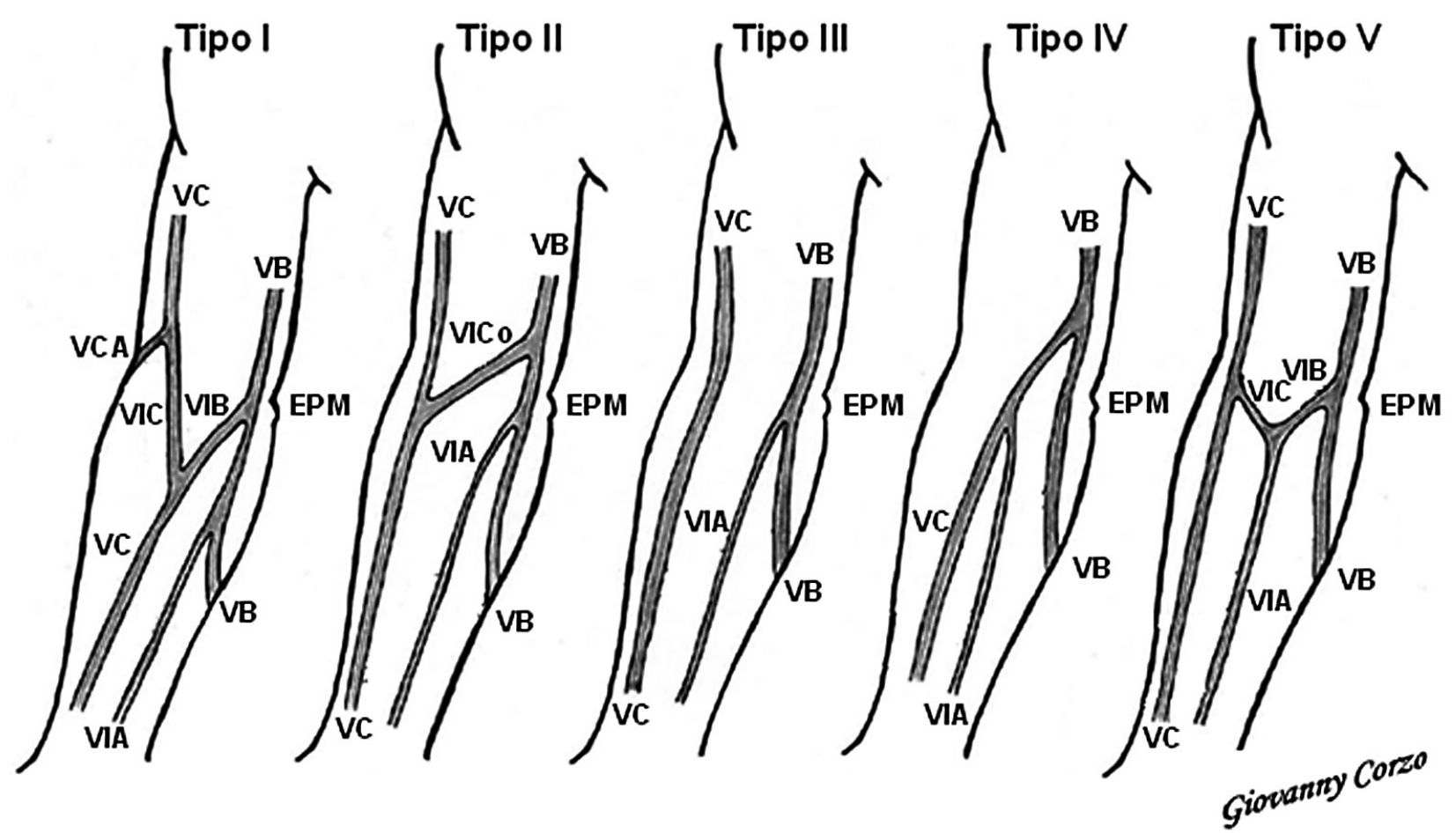

Fig. 1. Descripción de los patrones venosos de la fosa cubital según la propuesta de del Sol et al. (1988) y del Sol et al. (2007). VC: vena cefálica; VB: vena basílica; VCA: vena cefálica accesoria; VIC: vena intermedia cefálica; VIB: vena intermedia basílica; VIA: vena intermedia del antebrazo; VICo: vena intermedia del codo; EPM: epicóndilo medial.

Tipo II: la VC origina la vena intermedia del codo (VICo), que se une a la VB; no existe VCA, siendo la VICo de mayor calibre y la VIA drena en la VB.

Tipo III: no existe comunicación entre la VB y la VC a nivel de la fosa cubital; la VIA drena en la VB.

Tipo IV: la VC drena en la VB y la VIA drena en la VC.

Tipo V: la clásica $\mathrm{M}$ en donde la VIA se divide en una rama que se une a la VC y otra rama que se une a la VB.

Tipo VI: otros patrones diferentes (Fig. 1).

El análisis descriptivo se realizó para variables cualitativas nominales y ordinales mediante porcentajes, las cuantitativas con media y desviación estándar en el caso de variables con distribución normal y las no paramétricas u ordinales identificadas con número mediante mediana y rango intercuartil. Se comparó el género y el patrón venoso mediante la prueba de K. Wallis y chi cuadrado.

Consideraciones éticas. El proyecto fue aprobado como un estudio sin riesgo por el Comité de Ética de la Universidad Autónoma de Bucaramanga dentro del marco de aprobación del proyecto en la convocatoria interna institucional, con código 190017.

\section{RESULTADOS}

Descripción de la población. Durante el periodo de 20092010 se incluyeron 400 participantes, 200 mujeres y 200 hombres, con la condición de ser nacidos en Bucaramanga, distribuidos $60 \%$ estudiantes y trabajadores de la universidad Autónoma de Bucaramanga y $40 \%$ personas externas a la universidad. Todos los participantes recibieron la información concerniente al proyecto y firmaron un consentimiento informado.

Del total de personas evaluadas, en un $4 \%$ se encontró un desacuerdo entre los dos evaluadores en el momento de definir el patrón siendo necesario repetir el procedimiento y tomar la opinión de un tercer evaluador.

Se encontró que en los 800 miembros superiores estudiados el patrón que predominó fue el III, con 297 casos (37\%) seguido del patrón II con 190 casos (24\%) y del patrón I con 114 casos (14\%) (Fig. 2).

En el miembro superior derecho para ambos géneros el patrón más frecuente fue el III, con 141 casos (35\%), seguidos del patrón II 89 casos (22\%) y el patrón I con 58 casos (15\%). En el miembro superior izquierdo para ambos géneros el más frecuente fue el patrón III 156 casos 
(39\%), seguidos por el patrón II con 101 casos (25\%), y el patrón I con 56 casos (14\%) (Tabla I). No hay diferencias entre el patrón derecho o izquierdo en hombres o mujeres, Chi cuadrado 0.0679 y 0.728 valor de $\mathrm{p}(0,793$ y 0,39$)$ respectivamente.

El patrón II fue el más predominante en hombres (28\%), seguido del patrón III (21\%) y del I (20\%) en tanto que en mujeres predominó el patrón III (54\%) seguido del patrón II (19\%) y del I (10\%) (Tabla II). En el análisis de la frecuencia de la diferencia encontrada entre el patrón venoso II en hombres y el patrón III en mujeres fue estadísticamente significativa. Valor de p $(0,001)$.

En el miembro superior derecho en hombres, predominó el patrón II (25\%) seguido del patrón IV (23\%) y del I (20\%). En el miembro superior derecho en mujeres fue más frecuente el patrón III (51\%) seguido del II (20\%) y del VI (patrones no determinados previamente) con un $10 \%$ (Tabla II).

En el miembro superior izquierdo en hombres, predominó el patrón II (32\%) seguido del patrón III (21\%) y del I (19\%). En el miembro superior izquierdo en mujeres fue más frecuente el patrón III (57\%) seguido del II (18\%) y el I (9\%) (Tabla II).

El patrón venoso que en más ocasiones se presentó al mismo tiempo tanto en el lado derecho como en el izquierdo en la misma persona fue el patrón III con 94 personas equivalentes al 23,5\% seguido del patrón II con 45 personas (11\%) y el I con 22 personas (6\%), luego los patrones V y VI, ambos con $0,5 \%$ (Tabla III).

\section{DISCUSIÓN}

El patrón III fue el de mayor frecuencia en el presente estudio (37\%), también fue el mayor en miembros superiores derechos para ambos géneros (35\%), en miembros superiores izquierdos para ambos géneros (39\%), fue el más frecuente en mujeres (54\%) y el más frecuente en el miembro superior izquierdo en mujeres (57\%); en estos aspectos siempre estuvo seguido de los patrones II y I. También en el presente estudio, el patrón III fue más frecuente en el miembro superior derecho en mujeres (51\%), pero estuvo seguido de los patrones II y VI.

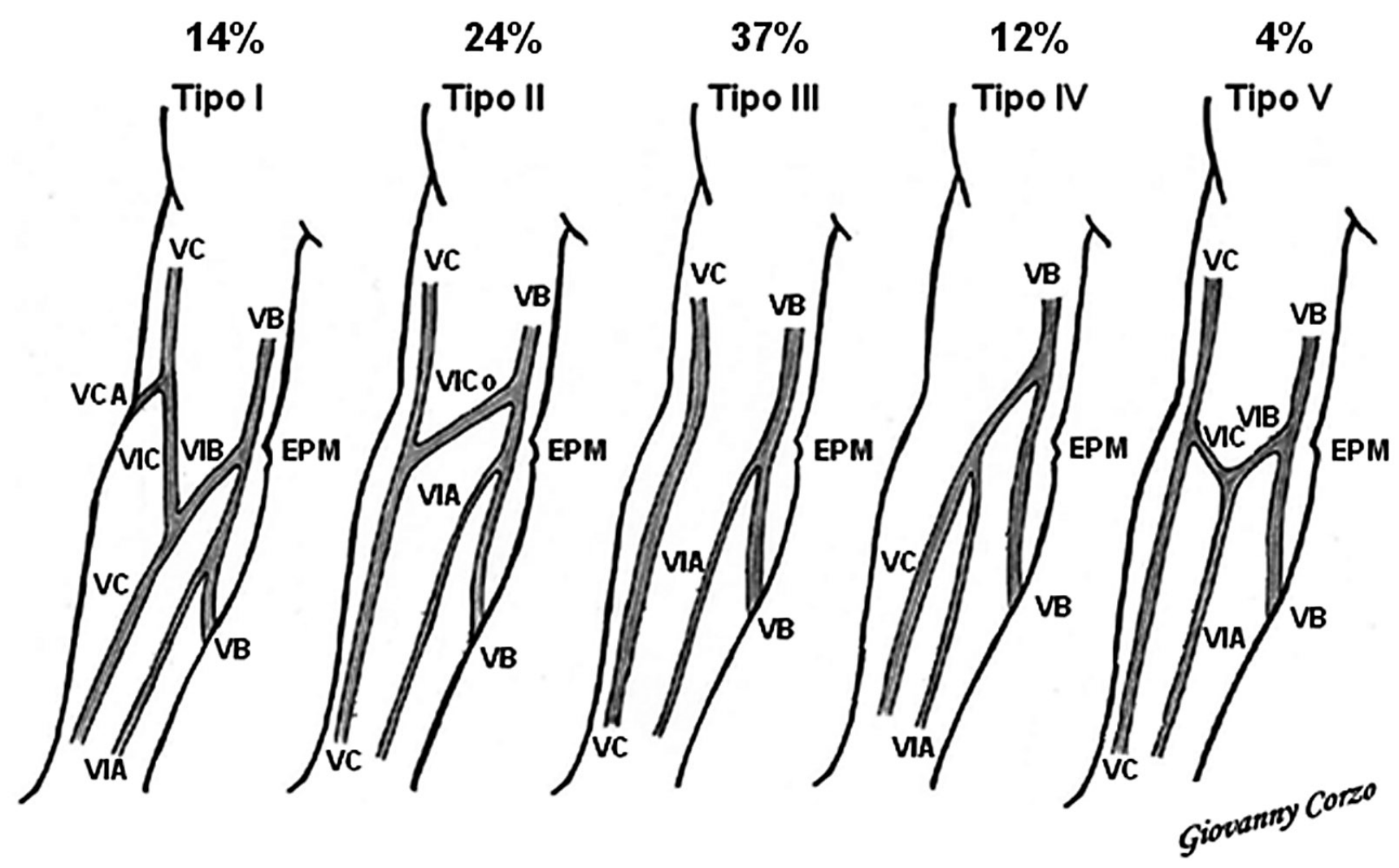

Fig. 2. Distribución de frecuencia de patrones venosos I, II, III y IV de la fosa cubital en una muestra de 400 individuos de ambos sexos, nacidos en Bucaramanga, Colombia. 
Tabla I. Distribución de frecuencia de patrones venosos de la fosa cubital de acuerdo al lado en una muestra de 400 individuos de ambos sexos, nacidos en Bucaramanga, Colombia. Abreviatura: MS: miembro superior.

\begin{tabular}{|c|c|c|c|c|c|c|}
\hline Patrón & $\begin{array}{l}\text { Miemb ro superior } \\
\text { Derecho } n=400\end{array}$ & $\%$ & $\begin{array}{c}\text { Miembro superior Izquierdo } \\
n=400\end{array}$ & $\%$ & Total & $\%$ \\
\hline I & 58 & 15 & 56 & 14 & 114 & 14 \\
\hline II & 89 & 22 & 101 & 25 & 190 & 24 \\
\hline III & 141 & 35 & 156 & 39 & 297 & 37 \\
\hline IV & 54 & 13 & 38 & 9 & 92 & 12 \\
\hline V & 15 & 4 & 16 & 5 & 31 & 4 \\
\hline VI & 43 & 11 & 33 & 8 & 76 & 9 \\
\hline Total & 400 & 100 & 400 & 100 & 800 & 100 \\
\hline
\end{tabular}

Tabla II. Distribución de frecuencia de patrones venosos de la fosa cubital de acuerdo al género en una muestra de 400 individuos de ambos sexos, nacidos en Bucaramanga, Colombia. Abreviaturas: MS: miembro superior MSS: miembros superiores

\begin{tabular}{|c|c|c|c|c|c|c|c|c|c|c|c|c|}
\hline \multirow{3}{*}{ Patrón } & \multicolumn{6}{|c|}{ Hombres } & \multicolumn{6}{|c|}{ Mujeres } \\
\hline & \multicolumn{2}{|c|}{ MS derecho } & \multicolumn{2}{|c|}{ MS izquierdo } & \multicolumn{2}{|c|}{ Total } & \multicolumn{2}{|c|}{ MS derecho } & \multicolumn{2}{|c|}{ MS izquierdo } & \multicolumn{2}{|c|}{ Total } \\
\hline & $\mathrm{N}$ & $\%$ & $\mathrm{~N}$ & $\%$ & N (200) & $\%$ & $\mathrm{~N}$ & $\%$ & $\mathrm{~N}$ & $\%$ & N (200) & $\%$ \\
\hline I & 41 & 20 & 38 & 19 & 79 & 20 & 17 & 9 & 18 & 9 & 35 & 10 \\
\hline II & 48 & 25 & 64 & 32 & 112 & 28 & 41 & 20 & 37 & 18 & 78 & 19 \\
\hline III & 39 & 19 & 42 & 21 & 81 & 21 & 102 & 51 & 114 & 57 & 216 & 54 \\
\hline IV & 46 & 23 & 28 & 14 & 74 & 18 & 8 & 4 & 10 & 5 & 18 & 4 \\
\hline $\mathrm{V}$ & 4 & 2 & 7 & 4 & 11 & 2 & 11 & 6 & 9 & 4 & 20 & 5 \\
\hline VI & 22 & 11 & 21 & 10 & 43 & 11 & 21 & 10 & 12 & 7 & 33 & 8 \\
\hline Total & 200 & 100 & 200 & 100 & 400 & 100 & 200 & 100 & 200 & 100 & 400 & 100 \\
\hline
\end{tabular}

Tabla III. Frecuencia de equivalencia del mismo patrón venoso en ambos miembros superiores para la misma persona. MSI: miembro superior izquierdo; MSD: miembro superior derecho.

\begin{tabular}{cccccccc}
\hline & Patrón MSI & & & & & \\
\cline { 2 - 7 } Patrón MSD & I & II & III & IV & V & VI & Total \\
\hline I & 22 & 14 & 5 & 6 & 2 & 9 & 58 \\
$\%$ & $\mathbf{6}$ & 29 & 1,2 & 1,5 & 0,5 & 2,25 & 14,5 \\
II & 11 & 45 & 23 & 0 & 4 & 6 & 89 \\
$\%$ & 3 & $\mathbf{1 1}$ & 6 & 0 & 1 & 1,5 & 22,25 \\
III & 6 & 14 & 94 & 13 & 6 & 8 & 141 \\
$\%$ & 1,5 & 3,5 & $\mathbf{2 3 , 5}$ & 3,25 & 1,5 & 0,02 & 35,25 \\
IV & 9 & 10 & 16 & 16 & 1 & 2 & 54 \\
$\%$ & 2,25 & 2,5 & 4 & $\mathbf{4}$ & 0,25 & 0,5 & 13,5 \\
V & 1 & 1 & 10 & 0 & 2 & 6 & 15 \\
$\%$ & 0,25 & 0,25 & 2,5 & 0,25 & $\mathbf{0 , 5}$ & 1,5 & 3,75 \\
VI & 7 & 17 & 8 & 3 & 1 & 2 & 43 \\
$\%$ & 1,75 & 4,25 & 0,02 & 0,75 & 0,25 & $\mathbf{0 , 5}$ & 10,75 \\
Total & 56 & 101 & 156 & 38 & 16 & 33 & 400 \\
$\%$ & 14 & 25,25 & 39 & 9,5 & 4 & 8,25 & 100 \\
\hline
\end{tabular}


Algunos autores han mostrado cifras de frecuencia muy bajas para el patrón III como es el caso de Berry \& Newton (1908) quienes reportaron sólo un 1\% de este patrón en individuos británicos, o los de Singh et al. con un $10 \%$ en personas nigerianas. Sin embargo otros autores han descrito cifras más altas: del Sol et al. encontraron un 17,3\% en individuos chilenos en 1990, mientras del Sol et al. señalan un 25\% en niños brasileños en 1988 y del Sol et al. (2007) encontraron un 24\% de este patrón en individuos del grupo étnico Mapuche en Chile; es de resaltar que en el grupo Mapuche el patrón III fue el segundo en frecuencia $(28,8 \%)$ en la fosa cubital izquierda para ambos géneros al igual que fue el segundo patrón en frecuencia en la fosa cubital izquierda tanto para mujeres $(28,3 \%)$ como para hombres (30\%). Es importante tener en cuenta que en nuestro estudio el patrón III no presentó una diferencia marcada en relación al lado (derecho 35\% e izquierdo 39\%), sin embargo si fue notable la diferencia por género, ya que en la totalidad de los hombres sólo presentaron patrón III el 21\% (por debajo de los patrones II con $28 \%$ y I con 20\%), en tanto que en la totalidad de las mujeres el patrón III fue el primero (54\%) bastante alejado del segundo en frecuencia, el patrón II (19\%), lo cual señala que la cifra de presentación de patrón III en el contexto global de este estudio depende en gran parte de su elevada cifra en mujeres.

En el presente estudio el patrón II que ocupó el segundo puesto en frecuencia (24\%), fue el predominante en hombres (28\%), mientras en mujeres estuvo en segundo lugar (19\%), fue el más frecuente en el miembro superior derecho en hombres (25\%), y el más frecuente en el miembro superior izquierdo en hombres (32\%). Nuestra cifra de patrón II es un poco más baja a la encontrada por del Sol et al. (1988) con 30\%, por del Sol et al. (1990) con $28,75 \%$, por del Sol et al. (2007) con 28,3\%. De forma diferente, Berry \& Newton reportaron valores elevados para este patrón con $83 \%$, al igual que Charles (1932) con $70 \%$, en tanto que Singh et al. mostraron un valor bajo de $11 \%$ en población nigeriana.

En nuestro estudio el patrón I se presentó en 14\% de los miembros superiores estudiados ocupando el tercer lugar de frecuencia global; este patrón y mantuvo una distribución similar para el lado derecho e izquierdo en el contexto global (15\%) y fue proporcional en hombres para ambos lados (20\%) y en mujeres para ambos lados (10\%). El patrón I, en términos generales ha sido señalado como el patrón predominante a nivel de la fosa cubital en estudios previos como los de Charles, 27,5\% en población Americana, del Sol et al. (1988) 30\% en niños brasileños, del Sol et al. (1990) 36,25\% en jóvenes chilenos, y del Sol et al. (2007) $38,7 \%$ en el grupo étnico Mapuche en donde predominó en el lado derecho en ambos géneros.
El patrón IV en nuestro estudio se encontró en un 12\% con mayor presentación hombres $(74 \%)$ con respecto a las mujeres (4\%). Fue mayor en el lado derecho (13\%) comparado con el izquierdo (9\%). También fue el segundo patrón en frecuencia en el lado derecho en hombres (23\%). De este patrón se han reportado cifras previas que van desde $0 \%$ por Okamoto, 1,6\% por Charles, 4,3\% por del Sol et al. (2007), $10 \%$ por Singh et al., en nigerianos, y $19,5 \%$ por Halim \& Abdi.

El patrón $\mathrm{V}$ que lo quisimos incluir en este estudio como un patrón independiente, en razón a que no habían datos importantes en nuestra población sobre la distribución clásica en "M" obtuvo una frecuencia de presentación de 4\%; su presentación fue levemente mayor en el lado derecho en mujeres (6\%). Estos resultados bajos son similares a los encontrados por del Sol et al. (1990) de 0,5\%, y al 1\% encontrado por del Sol et al. (2007) en el grupo étnico Mapuche en Chile. Okamoto encontró un $1 \%$ de esta distribución en japoneses y Skewes 1,7\%. Halim \& Abdi reportaron un 6,5\% en hindúes. Los valores descritos por estos autores se contraponen con los hallazgos de Paturet quien describe esta distribución venosa en el $60 \%$ de los casos o Singh et al. quienes informan un $40 \%$ de hallazgo en un estudio de 300 personas nigerianas.

Nuestros resultados en relación al patrón $\mathrm{V}$ nos hace coincidir con la idea planteada por del Chiarugi (1912) y por del Sol et al. $(1988,1990,2007)$ en donde en muchos casos en donde se mostró una gran frecuencia de "M" clásica, quizá la vena que se dividía en venas intermedia cefálica e intermedia basílica no era la vena intermedia del antebrazo sino que la división obedecía a la vena cefálica y la que se estaba considerando como cefálica en la "M" clásica realmente era la vena cefálica accesoria. Con esto se apoya la idea de que el llamado patrón clásico en "M" no sea considerado un patrón como tal sino que se estudie como una variación anatómica de baja frecuencia de hallazgo.

El patrón VI de nuestro estudio estuvo presente en un 9\%, siendo mayor en el lado derecho (11\%) comparado con el izquierdo (4\%); en general su presentación fue similar para ambos lados en los dos géneros (11\%) excepto en el lado izquierdo en mujeres en donde su presentación fue un poco más baja $(7 \%)$.

El patrón venoso que en más ocasiones se presentó al mismo tiempo tanto en el lado derecho como en el izquierdo en la misma persona fue el patrón III con un $23.5 \%$ seguido del patrón II con $11 \%$ y el I con $6 \%$, luego los patrones $\mathrm{V}$ y $\mathrm{VI}$, ambos con $0,5 \%$. Ver tabla III. No encontramos datos previos para comparar esta situación pero sería interesante a futuro saber si alguno de los patrones tiene una predisposición significativa a presentarse con mayor frecuencia en ambos 
lados en la misma persona lo cual tendría un potencial uso en la parte de pediatría en donde en muchos casos la visibilidad de las venas es muy pobre.

En conclusión, en sentido general, el patrón que predominó fue el III pero determinado por su alta frecuencia en mujeres. En hombres predominó el patrón II. Se confirma la baja presencia de patrón clásico en "M" con lo cual se sustenta la idea de que él no sea un patrón sino una variación anatómica. El patrón que en más ocasiones se presentó al mismo tiempo en ambos lados para la misma persona fue el III.

\section{AGRADECIMIENTOS}

A la Universidad Autónoma de Bucaramanga, en cuya Facultad de Salud recibimos toda la colaboración y ayuda técnica para la realización del presente trabajo.

CORZO, G. E. G; GÓMEZ, D. O. L.; NIÑO, M. M. E.; REY, T. R. J. \& PEDRAZA, D. L. J. Distribution pattern of the veins of the cubital fossa in a sample of people born in Bucaramanga, Colombia. Int. J. Morphol., 28(4):1011-1018, 2010.

SUMMARY: The rational use of access to the superficial veins in the cubital fossa is particularly important in pediatric patients, with fistulas for dialysis, chemotherapy, or central venous access routes. Several venous patterns have been described for their benefits or risks. The prevalence of venous patterns in a population of both sexes born in Bucaramanga, Colombia, in 800 upper limbs (200 men and 200 women), according to the classification proposed by del Sol et al. $(1988,2007)$. The descriptive analysis for nominal and ordinal qualitative variables with percentages, the quantitative mean and standard deviation for normally distributed variables and nonparametric identified by number using median and interquartile range, was compared by gender and venous pattern through test and chi square Kwallis. The predominant pattern was III, 297 cases (37\%) determined by a high frequency in women (54\%), followed by II 190 cases (24\%). On the right side for both sexes the most common pattern was III, 141 cases (35\%), followed by II, 89 cases (22\%). On the left side for both genders predominant pattern III, 156 cases (39\%), followed by the second with 101 cases $(25 \%)$. Pattern II was greater in men (28\%), followed by III (21\%). In women the pattern III predominated, followed by II (19\%). On the right side in men, II predominated (24\%) followed by IV (23\%). On the right side III predominated in women (51\%) followed by II (20\%). On the left side in men, the predominant pattern II (32\%) followed by III $(21 \%)$. On the left side in women predominated III (57\%) followed by II (18\%). The pattern appeared more often at the same time on both sides in the same person was III $(16.6 \%)$.

KEY WORDS: Venipuncture; Veins; Cubital fossa.

\section{REFERENCIAS BIBLIOGRÁFICAS}

Abrams, R. A; Brown, R. A. \& Botte, M. J. The superficial branch of the radial nerve: an anatomic study with surgical implications. J. Hand Surg., 17:1037-41, 1992.

Auerbach, D. M.; Collins, E. D.; Kunkle, K. L. \& Monsanto E. H. The radial sensory nerve: an anatomic study. Clin. Orthop., 308:241-9, 1994.

Berry, R. J. A. \& Newton, H. A. S. A study of the superficial veins of the superior extremity in 300 living subjects. Anat. Anz., 33(5):591-601, 1908.

Boeson, M. B.; Hranchook, A. \& Stoller, J. Peripheral nerve injury from intravenous cannulation: a case report. $A A N A$ $J, 68: 53-7,2000$.

Charles, C. M. On the arrangement of the superficial veins of the cubital fossa in American white and American negro males. Anat. Rec, 54:9-14, 1932.
Chiarugi, G. Istituzioni di Anatomía dell'Uomo. Milano, Societa Editrice Libraría, 1912.

Del Sol, M.; De Angelis, M. A. \& Bolini, P. A. D. Formações venosas na fossa cubital da criança. Pediatría Moderna, 23(4):225-31, 1988.

Del Sol, M.; Mardones, L. M. \& Bustos, T. E. Formaciones Venosas de la Fosa Cubital en el Individuo Mapuche. Estudio Bioscópico. Int. J. Morphol, 25(4):885-94, 2007.

Del Sol, M.; Wuster, A. \& Fritz, I. Tipos de formaciones venosas en la fosa cubital en jóvenes chilenos. Rev. Chil. Tecnol. Méd., 13(2):646-9, 1990.

Halim, A. \& Abdi, S. H. M. Superficial venous patterns in the cubital region of Indians. Anat. Rec., 178(3):631-6, 1974. 
CORZO, G. E. G; GÓMEZ, D. O. L.; NIÑO, M. M. E.; REY, T. R. J. \& PEDRAZA, D. L. J. Distribución de los patrones venosos de la fosa cubital en una muestra de personas nacidas en Bucaramanga, Colombia. Int. J. Morphol., 28(4):1011-1018, 2010.

Horowitz, S. H. Venipuncture-induced neuropathic pain: the clinical syndrome, with comparisons to experimental nerve injury models. Pain, 94:225-9, 2001.

Lirk, P.; Keller, C.; Colvin, J.; Colvin, H.; Rieder, J.; Maurer, H. \& Moriggl B. Unintentional arterial puncture during cephalic vein cannulation: case report and anatomical study. Br. J. Anaesth., 92(5):740-2, 2004.

Okamoto, K. A study of the superficial veins in the superior extremity of live Japanese. Anat. Rec., 23:323-33, 1922.

Paturet, G. Traite d'anatomie humaine. Paris, Masson, 1951. Rodriguez-Niedenfuhr, M.; Vazquez, T. \& Nearn, L. Variations of the arterial pattern in the upper limb revisited: a morphological and statistical study, with a review of the literature. J. Anat., 199:547-66, 2001.

Singh, S. P.; Ekandem, G. J. \& Bose, E. S. A study of the superficial veins of the cubital fossa in Nigerian subject. Acta Anat., 774:311-20, 1982.

Skewes, E. Estudio de las venas superficiales del antebrazo en los chilenos. Bol. Soc. Biol., 19:75-81, 1944.

Stahl, S.; Kaufman, T. \& Ben-David, B. Neuroma of the superficial branch of the radial nerve after intravenous cannulation. Anesth. Analg., 83:180-2, 1996.

Thrush, D. N. \& Belsole, R. Radial nerve injury after routine peripheral vein cannulation. J. Clin. Anesth., 7:160-2, 1995.

Vialle, R. L.; Vialle, C. P.; Cronier, P.; Brillu, C.; Villapadierna, F. \& Mercier, P. Anatomic relations between the cephalic vein and the sensory branches of the radial nerve: how can nerve lesions during vein puncture be prevented? Anesth. Analg., 93:1058-61, 2001.

Yamada, K.; Yamada, K. E.; Katsuda I. \& Hida, T. Cubital fossa venipuncture sites based on anatomical variations and relationships of cutaneous veins and nerves. Clin. Anat., 21(4):307-13, 2008.
Dirección para correspondencia: Edgar Giovanni Corzo Gómez Universidad Autónoma de Bucaramanga Calle 157 número 19-55

Canaveral

Parque Floridablanca Santander COLOMBIA

Email: ecorzo@unab.edu.co

Recibido : 12-07-2010

Aceptado: 11-09-2010 